\title{
SISTEMAS DE INFORMACIÓN GEOGRÁFICA PARA UNIDADES DE INFORMACIÓN
}

\author{
Estefanía Aguilar-Moreno y Carlos Granell-Canut
}

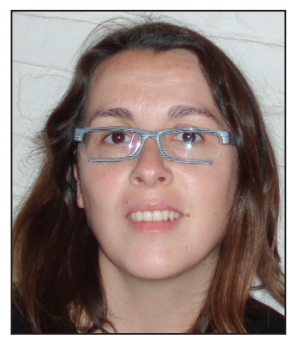

Estefanía Aguilar-Moreno es licenciada en documentación y máster en sociedad de la información y el conocimiento por la Universitat Oberta de Catalunya (UOC). Trabaja en temas relacionados con la inteligencia competitiva y colabora como consultora en los Estudios de Ciencias de la Información y de la Comunicación de la UOC. Actualmente cursa los estudios de doctorado en el programa de la Sociedad de la Información y el Conocimiento en IN3-UOC.

http://orcid.org/0000-0002-3157-2015

Universitat Oberta de Catalunya. IN3 Roc Boronat, 117. 08018 Barcelona, España eaguilarm@uoc.edu

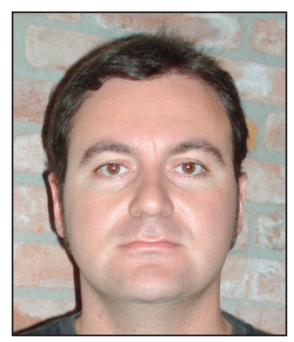

Carlos Granell-Canut, doctor en geoinformática por la Universitat Jaume I, es investigador postdoctoral en el Instituto de Medio Ambiente y Sostenibilidad del Joint Research Centre (JRC) de la Comisión Europea. Su investigación se centra en la aplicación de los sistemas de información geográfica y las tecnologías geoespaciales. https://orcid.org/0000-0003-1004-9695

European Commission - Joint Research Centre, Institute for Environment and Sustainability Via Enrico Fermi, 2749. 21027 Ispra, Italia carlos.granell@jrc.ec.europa.eu

\section{Resumen}

Se muestran los sistemas de información geográfica (Geographic Information Systems, GIS) como tecnología de soporte a la toma de decisiones en bibliotecas y unidades de información. Se presenta el concepto de GIS y sus sinergias con las unidades de información a partir de la revisión de diferentes proyectos que los utilizan en bibliotecas. Finalmente se apuntan diferentes contextos relacionados con unidades de información en los que se pueden aplicar GIS para la mejora de servicios y productos por parte de profesionales de la información.

\section{Palabras clave}

Sistemas de información geográfica, GIS, Unidades de información, Visualización de datos, Análisis de datos, Servicios geográficos en bibliotecas.

\section{Title: Geographic information systems for information units}

\begin{abstract}
The aim of the paper is to serve as a gateway to the world of geographic information systems (GIS), seen as technologies to support decision-making services in libraries and information units. Through the review of various projects that make use of GIS technologies in libraries, the concept of GIS and its synergies with information units is introduced. Finally, the paper points out different contexts and situations, in relation to information units, in which GIS could be used to improve services and products by information professionals.
\end{abstract}

\section{Keywords}

Geographic information systems, GIS, Information units, Data visualization, Data analysis, Geographic services in libraries.

Aguilar-Moreno, Estefanía; Granell-Canut, Carlos (2013). "Sistemas de información geográfica para unidades de información". El profesional de la información, enero-febrero, v. 22, n. 1, pp. 80-86.

http://dx.doi.org/10.3145/epi.2013.ene.11 


\section{Introducción}

En 1995 The journal of academic librarianship ${ }^{1}$ publicó un número especial sobre sistemas de información geográfica (en adelante GIS, por sus siglas en inglés) y bibliotecas. Desde los años 90 la bibliografía recoge estudios sobre uso y aplicación de GIS en unidades de información, principalmente en Estados Unidos, Canadá y Reino Unido. Sin embargo, a pesar de que han transcurrido ya casi 20 años desde el inicio de su aplicación en bibliotecas, archivos y centros de documentación, estos sistemas no acaban de extenderse entre el colectivo de profesionales de la información españoles ${ }^{2}$.

Autores como Allen (2001), Michalec y Welsh (2007), Bishop y Mandel (2010), han analizado las publicaciones sobre GIS y bibliotecas, pero no es objeto de este trabajo estudiar lo publicado sobre este tema, sino impulsar su uso en unidades de información como herramientas para la creación de nuevos servicios, optimización de recursos y soporte a la toma de decisiones. Se muestran diferentes usos y aplicaciones de GIS a partir de las referencias existentes en la literatura especializada, y se hacen algunas propuestas.

\section{¿Qué es un GIS?}

Los mapas son posiblemente los tipos de datos geográficos más familiares para cualquier usuario. Sin embargo, los datos de la red de carreteras, las parcelas catastrales, los ríos o los límites administrativos (provincias, comunidades) son también datos geográficos, con independencia de que se representen o no sobre un mapa. La característica común a todos es que contienen una referencia a una posición o localización concreta, explícita (como coordenadas geográficas) o implícita (código postal, nombre de una calle). Desde la perspectiva de un usuario no experto, GIS se entiende como un conjunto de programas o aplicaciones de ordenador para la creación, tratamiento, análisis y visualización de datos geográficos (Cox; Gifford, 1997).

Una característica diferenciadora de las aplicaciones GIS es la gestión de los datos geográficos mediante capas temáticas que se superponen a un mapa (figura 1). Por ejemplo, la capa de carreteras de una provincia estará formada por las entidades geográficas (líneas) que representan cada carretera junto con los atributos asociados a cada entidad geográfica (tipo de carretera, año de construcción, etc.). Así, con un vistazo a un mapa de una ciudad con las capas de la ubicación de las bibliotecas y de la densidad de población, podríamos obtener una idea bastante nítida de la necesidad o no de una nueva biblioteca en un barrio determinado sin recurrir a análisis complejos. Es decir, con una perspectiva espacial se hacen visibles relaciones que no se aprecian observando los datos de forma directa.

Un GIS es un conjunto de aplicaciones informáticas para la creación, tratamiento, análisis y visualización de datos geográficos

Los usuarios por excelencia de los GIS han sido tradicionalmente las administraciones públicas, ya que son las encargadas de la gestión de recursos con fuerte dependencia del territorio, como parques naturales, catastros, usos del suelo o urbanismo. Se calcula que el $80 \%$ de los datos de la administración pública contiene explícitamente referencias geográficas (Hawkins, 1994). Sin embargo el concepto GIS no debería acotarse a un programa específico que permite realizar operaciones y análisis sobre datos geográficos, sino que debe considerarse como un conjunto de herramientas, programas y tecnologías de diversa índole que ofrecen una gran variedad de funciones y posibilidades para el tratamiento de la información geográfica. Las tecnologías geoespaciales pueden adaptarse a multitud de escenarios y problemas con diferentes grados de complejidad, como veremos a continuación.

Los GIS resultan atractivos en diversos contextos ya que la dimensión geográfica y la posición son conceptos transversales a muchas disciplinas. Asimismo, una primera incursión en GIS no implica una curva de aprendizaje lenta y compleja para obtener los primeros resultados, pues existen programas y tecnologías geoespaciales centradas en tareas particulares muy asequibles. Muchos programas GIS son libres $u$ open source (Morris, 2006; Abresch et al., 2008; Neatrour et al., 2011) y se pueden utilizar en el contexto de una biblioteca con coste cero o mínimo. Por lo tanto, existen pocas barreras para el uso de GIS, tanto económicas como técnicas, y en cambio ofrecen grandes ventajas.

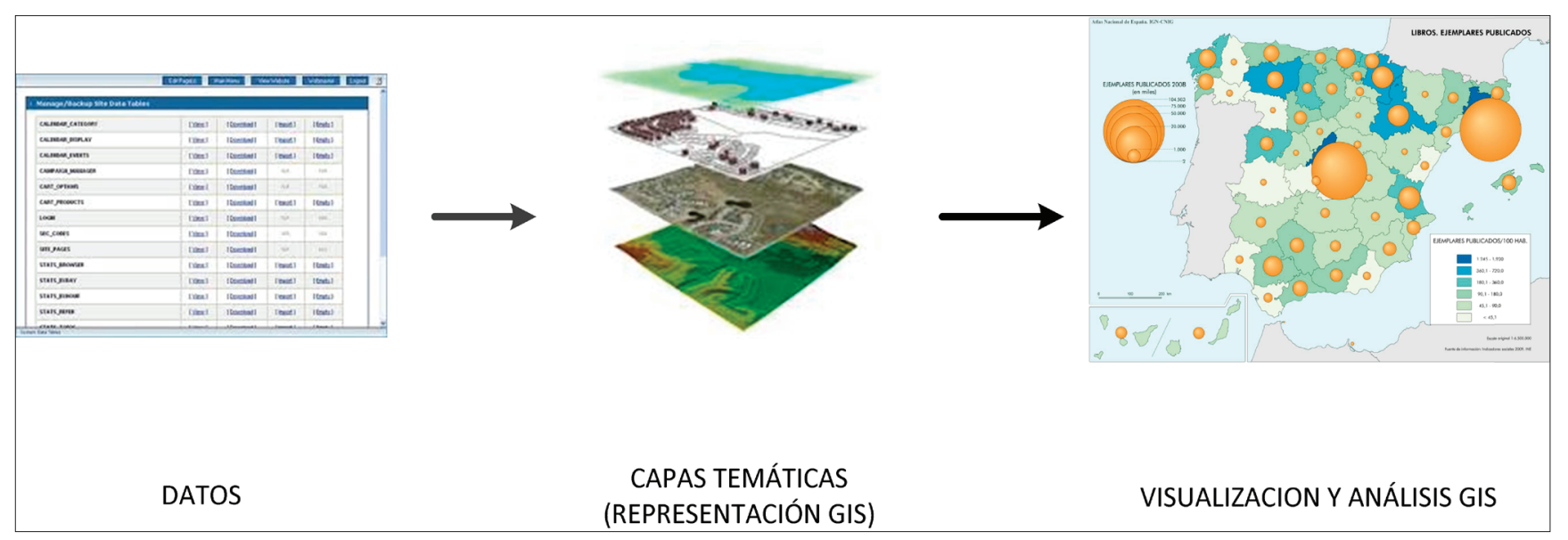

Figura 1. Flujo de actividades simplificado en un GIS de capas temáticas georreferenciadas 


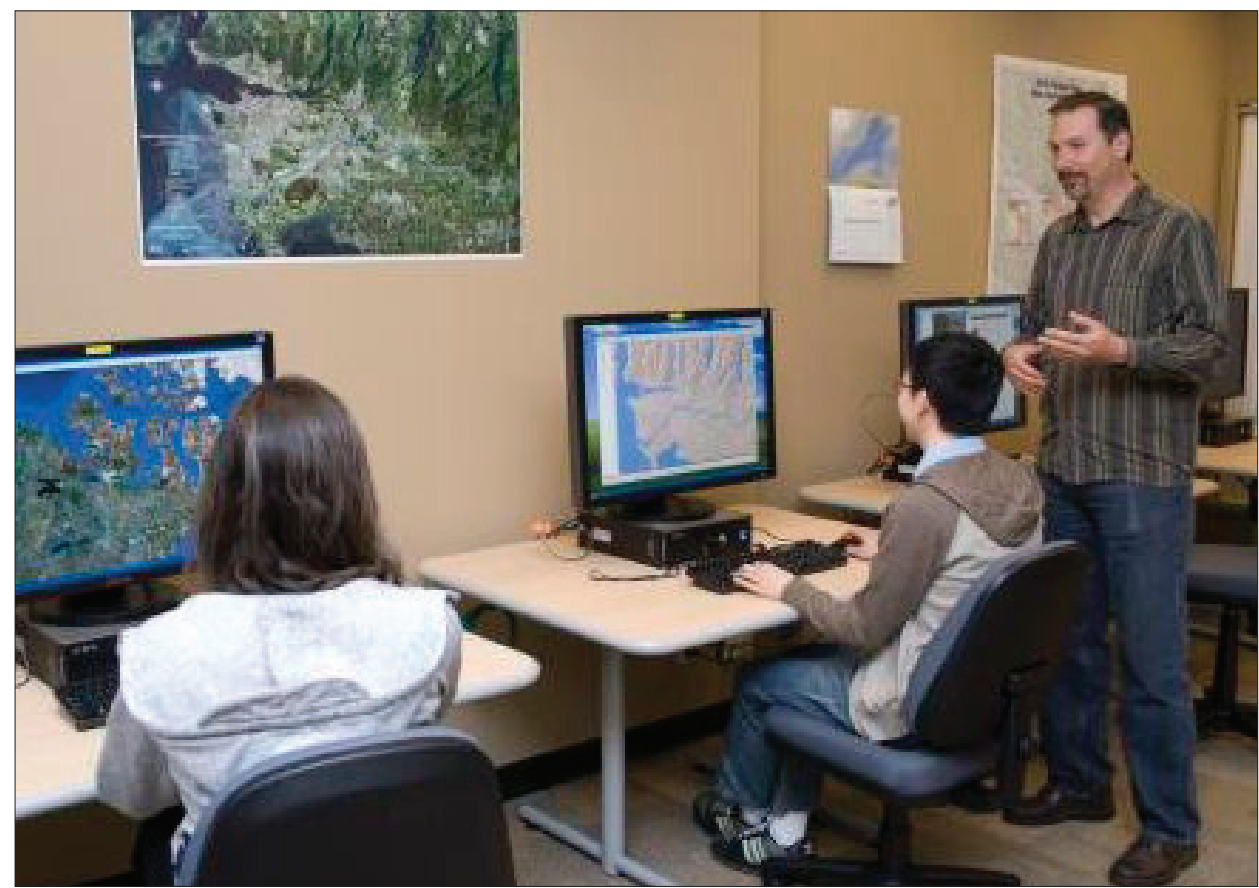

Figura 2. El bibliotecario de la University of British Columbia asesora sobre cómo integrar datos geográficos en proyectos de investigación. http://guides.library.ubc.ca/gis

\section{Usos de GIS en bibliotecas}

Un primer aspecto que hace interesante la utilización de GIS en centros bibliotecarios es que sus datos contienen de forma natural y explícita referencias geográficas. Por ejemplo, el registro de un usuario contiene un código postal, dirección postal o nombre de ciudad. Un libro del catálogo está publicado en una ciudad o su contenido puede versar sobre la historia de una región, ciudad o país. Es decir, las bibliotecas ya disponen de datos con claras referencias geográficas, por lo que GIS se convierte en una potencial herramienta para su explotación y visualización.

En segundo lugar las bibliotecas disponen de datos estructurados que facilitan y hacen viable su integración y representación mediante GIS (Kowal, 2002). Tanto las bibliotecas como GIS comparten una larga historia en estandarización de metadatos, en ocasiones con especificaciones y normas muy afines (Abresch et al., 2008), por lo que su explotación desde la perspectiva GIS no debería implicar en principio grandes modificaciones.

En la tabla 1 se señalan aplicaciones y usos de GIS en unidades de información ${ }^{3}$. Las principales se centran en tres aspectos:

- toma de decisiones en bibliotecas;

- interfaces de búsqueda;

- GIS como recurso bibliotecario.

Adicionalmente se destacan los trabajos que han hecho un uso combinado de datos disponibles en bibliotecas y de otros facilitados por organismos públicos.

Los medios habituales de presentar las estadísticas de bibliotecas son las hojas de cálculo, tablas y gráficas; sin embargo los GIS abren nuevas posibilidades más intuitivas e innovadoras de representación de datos estadísticos complejos (Coyle, 2011).
En algunos trabajos se usan GIS para realizar estudios sobre gestión, localización, desarrollo y uso de colecciones (Hawkins, 1994), así como para detectar puntos calientes de circulación de ejemplares dentro de una biblioteca (Coyle, 2011). Una posible mejora de la colección puede ser disponer los materiales según el origen de los usuarios, por ejemplo comunidades que comparten una misma lengua o personas con deficiencias visuales (Venuda, 2005).

Un GIS puede ser útil también para el diseño interior de espacios en bibliotecas (Xia, 2004), para el seguimiento del movimiento de usuarios dentro de las dependencias (Xia, 2005), para determinar la necesidad de sistemas de videovigilancia en zonas con puntos negros en la desaparición de materiales, o para trasladar éstos a zonas más visibles (Coyle, 2011).

Sin embargo, la mayor parte de los trabajos revisados en este artículo recogen aplicaciones para la visualización de datos bibliotecarios que ayudan a la toma de decisiones sobre la apertura, cierre o reubicación de bibliotecas (Preiser; Wang, 2006; Hertel; Sprague, 2007; Venuda, 2005). Mediante los códigos postales (o direcciones) de los usuarios, se puede utilizar un GIS para situar o eliminar nuevos centros bibliotecarios considerando la proximidad de estos servicios a los usuarios reales o potenciales, o incluso averiguando el nivel de solapamiento con la red de bibliotecas existente (Coyle, 2011). También se pueden analizar las áreas de la ciudad y las bibliotecas que tienen más libros prestados. Otro buen ejemplo es el tráfico de materiales de préstamo interbibliotecario, que puede ayudar a ajustar los materiales disponibles en una red de bibliotecas. Según Preiser y Wang (2006), con la aplicación de GIS se amplía el concepto de biblioteca como centro de conocimiento al servicio de una comunidad de usuarios. Las bibliotecas como lugares de participación, educación o entretenimiento, así como su oferta de servicios como acceso a internet, también pueden ser objeto de estudio de GIS.

Hawkins (1994) habla de la puesta en valor de los datos de encuestas de satisfacción a través de GIS. Incluyendo en el formulario de encuesta algún dato que pueda localizar al usuario geográficamente, se conectarán las respuestas de la encuesta con otras informaciones demográficas del usuario interesantes para mejorar los servicios.

GIS también puede dar apoyo en el diseño de las plantillas personal de las bibliotecas (Preiser; Wang, 2006). Hacer estimaciones sobre el número de potenciales usuarios de cada servicio, sus perfiles, franjas horarias en las que hacen 


\begin{tabular}{|c|c|c|c|c|c|c|c|c|c|c|c|c|}
\hline \multirow[b]{2}{*}{ Aplicaciones } & \multirow[b]{2}{*}{ Usos } & \multicolumn{11}{|c|}{ Autores } \\
\hline & & 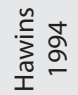 & 仓ั & 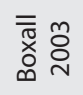 & 赵家 & $\stackrel{\pi}{\times} \stackrel{n}{\stackrel{0}{0}}$ & 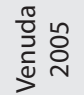 & 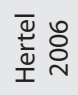 & 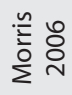 & 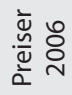 & 을 & ฮั่ \\
\hline \multirow{15}{*}{$\begin{array}{l}\text { Visualización } \\
\text { de información } \\
\text { para la toma de } \\
\text { decisiones en } \\
\text { bibliotecas }\end{array}$} & Préstamos & $\mathrm{x}$ & & & & & & & & $\mathrm{x}$ & & $\mathrm{x}$ \\
\hline & $\begin{array}{l}\text { Gestión y } \\
\text { organización de } \\
\text { colecciones }\end{array}$ & $\mathrm{x}$ & & & & & & & & & & $x$ \\
\hline & Uso de colecciones & $\mathrm{x}$ & & & & & & & & & & $\mathrm{x}$ \\
\hline & $\begin{array}{l}\text { Desarrollo de } \\
\text { colecciones }\end{array}$ & $\mathrm{x}$ & & & & & & & & & & $x$ \\
\hline & $\begin{array}{l}\text { Localización física de } \\
\text { materiales }\end{array}$ & & & & & & & & & & & $\mathrm{x}$ \\
\hline & $\begin{array}{l}\text { Toma de decisiones } \\
\text { (mantenimiento, } \\
\text { apertura, cierre, } \\
\text { localización) }\end{array}$ & $x$ & & & & & $x$ & $x$ & & $x$ & & $x$ \\
\hline & Tipo de usuarios & $x$ & & & & & $x$ & & & & & \\
\hline & $\begin{array}{l}\text { Movimiento de } \\
\text { usuarios en la } \\
\text { biblioteca }\end{array}$ & & & & & $\mathrm{x}$ & & & & & & \\
\hline & $\begin{array}{l}\text { Encuestas de } \\
\text { satisfacción }\end{array}$ & $\mathrm{x}$ & & & & & $\mathrm{x}$ & & & & & \\
\hline & Diseño de espacios & $\mathrm{x}$ & & & $\mathrm{x}$ & $\mathrm{x}$ & & & & $\mathrm{x}$ & & \\
\hline & $\begin{array}{l}\text { Potenciación de } \\
\text { servicios }\end{array}$ & $x$ & & & & & $\mathrm{x}$ & & & & & \\
\hline & Marketing de servicios & $x$ & & & & & $x$ & & & & & \\
\hline & $\begin{array}{l}\text { Diseño de la plantilla } \\
\text { debibliotecarios }\end{array}$ & & & & & & & & & $\mathrm{x}$ & & \\
\hline & $\begin{array}{l}\text { Sistema de } \\
\text { videovigilancia }\end{array}$ & & & & & & & & & & & $\mathrm{x}$ \\
\hline & Bibliobús & $x$ & & & & & & & & & $x$ & \\
\hline $\begin{array}{l}\text { Interfaces de } \\
\text { búsqueda }\end{array}$ & $\begin{array}{l}\text { Criterios espaciales de } \\
\text { búsqueda }\end{array}$ & & $x$ & & & & & & $x$ & & & \\
\hline & $\begin{array}{l}\text { Suministro de datos } \\
\text { cartográficos en } \\
\text { sustitución del papel }\end{array}$ & $x$ & $x$ & & & & $x$ & & $x$ & & & \\
\hline $\begin{array}{l}\text { Incorporar datos } \\
\text { GIS como recurso }\end{array}$ & $\begin{array}{l}\text { Centro de datos } \\
\text { geográficos }\end{array}$ & & $x$ & $x$ & & & $\mathrm{x}$ & & & & & \\
\hline bibliotecario & $\begin{array}{l}\text { Asistencia en el } \\
\text { uso, localización y } \\
\text { enseñanza del uso de } \\
\text { datos GIS }\end{array}$ & & $\mathrm{x}$ & & & & & & & & & \\
\hline
\end{tabular}

Tabla 1. Aplicaciones y usos de GIS en unidades de información

uso de la biblioteca, etc., ayudará a definir mejor las necesidades organizativas de la plantilla.

Las bibliotecas móviles o bibliobuses parecen el servicio más obvio a tratar desde la perspectiva de GIS debido a su uso extensivo en análisis de trayectos; sin embargo sólo hay un trabajo en español (Castro-González; González-Gallardo, 2009) sobre un uso práctico para la definición de rutas de bibliotecas móviles y de las paradas más óptimas.

Pasando a un ámbito más tecnológico, los GIS ofrecen una nueva concepción de las interfaces de búsqueda. Cox y Gifford (1997) ya dejaron entrever la capacidad de las interfaces de usuario basadas en mapas como más intuitivas para la búsqueda de información en catálogos. Un usuario puede complementar criterios de búsqueda tradicionales (palabras claves o materias) con otros criterios espaciales. Asimismo, las búsquedas pueden acotarse a una determinada área o zona geográfica de interés identificada sobre un mapa. A modo de ejemplo, este tipo de interfaces permitirían la búsqueda de recursos sobre cierta materia editados en España o por autores nacidos en una área concreta indicada por el usuario.

Algunos trabajos hablan de la incorporación de GIS como recurso bibliotecario ${ }^{4}$ (figura 2). Hawkins (1994) recogía la posibilidad de suministrar los datos cartográficos en formato digital, en sustitución del papel. Morris (2006) se pregunta 
cómo pueden las bibliotecas académicas promocionar y facilitar el acceso a recursos GIS vía servicios web geográficos, por ejemplo para enlazar catálogos y registros de información geográfica con catálogos bibliotecarios. Cox y Gifford (1997), Boxall (2003), y Venuda (2005) se interesan por situar a las bibliotecas como centro de datos geográficos donde se localice, organice y suministre este tipo de información. Cox y Gifford (1997) opinan que las unidades de información deben prestar asistencia en el uso y enseñanza de GIS, y que la biblioteca puede ser un centro de generación de proyectos GIS por ser centro de referencia y lugar de encuentro de investigadores de varias disciplinas.

Por último, dentro de esta clasificación se recoge el uso combinado de datos públicos y datos de bibliotecas. En el año 2000 la publicación del censo de población de Estados Unidos marcó un hito en el uso de información pública desde la óptica de las bibliotecas. Muchas bibliotecas americanas emprendieron estudios sobre los usos y beneficios de dichos datos, llegando muchas a mejorar sus servicios de marketing mediante, por ejemplo, estudios demográficos de mercado o de demanda. Los GIS atrajeron inmediatamente la atención como herramientas especializadas en la creación de capas temáticas de datos demográficos visualizadas sobre mapas (Hertel; Sprague, 2007). Destaca el caso de The Public Library Geographic Database $^{5}$ (Koontz et al., 2004) que recoge datos de 17.000 bibliotecas públicas de Estados Unidos, en combinación con datos del censo.

Muchas de estas aplicaciones ya se están utilizando de forma habitual en bibliotecas, principalmente en el ámbito anglosajón. No obstante, aún hay camino por recorrer y GIS abre nuevas oportunidades a las bibliotecas para que actúen de manera proactiva a través de la generación y/o mejora de nuevos servicios.

\section{Nuevos usos y mejoras GIS para unidades de información}

Existen trabajos sobre la reestructuración de sistemas bibliotecarios ajustando la accesibilidad a las bibliotecas en función de sus usuarios reales y potenciales, mediante la apertura o cierre de bibliotecas. Sin embargo, no se cita la posibilidad de utilizar GIS desde una nueva perspectiva, por ejemplo para sustituir bibliotecas físicas por opciones móviles (bibliobus, préstamo a distancia) o virtuales. Dentro de este grupo, otro uso relacionado más directamente con el diseño de espacios y edificios podría ser de ayuda al esta- blecimiento de planes de emergencia a través de la correcta y rápida localización de aquellos materiales más valiosos, en caso de inundación o incendio, por ejemplo.

En segundo lugar, llama la atención la falta de sinergias entre aplicaciones GIS y estudios cienciométricos. La creación de mapas temáticos para la exploración visual de este tipo de estadísticas sobre producción científica y colaboración podría abrir nuevas perspectivas en este campo. Sumando la dimensión temporal y geográfica a los datos numéricos sobre publicaciones en forma de gráficas y tablas ${ }^{6}$ que viene facilitándose hasta el momento, daríamos lugar a la creación de atlas ${ }^{7}$ interactivos con un enfoque geográfico-temporal sobre la producción cienciométrica que podría, entre otros aspectos, ilustrar enormemente la colaboración entre autores.

El desarrollo de interfaces de búsqueda con herramientas GIS debe revisarse con atención. Por ejemplo, en bibliotecas universitarias con diferentes puntos de acceso dispersos en el campus, el sistema nos puede indicar visualmente dónde se localiza el ejemplar más próximo a nuestra ubicación. Este tipo de aplicaciones tiene una proyección más clara en dispositivos móviles, ya que la interfaz de búsqueda puede adaptarse y sincronizarse continuamente con la posición de usuario en cada momento. También es útil su uso en consorcios de bibliotecas, optimizando así la localización y envío de materiales por préstamo interbibliotecario.

Otra utilización es la relacionada con sistemas de inteligencia competitiva, especialmente en su vertiente de inteligencia territorial ${ }^{8}$. Parece evidente la conexión entre la toma de decisiones y el diseño de estrategias para el desarrollo de un territorio desde el prisma de las herramientas GIS. Se podrían mostrar relaciones espaciales entre actores que for- 
men parte del sistema de inteligencia, o la combinación de datos sobre recursos económicos, humanos y materiales, en contraste con el desarrollo económico y empresarial. Si bien se ha localizado alguna experiencia sobre la aplicación de mapas en la representación de este tipo de informaciones ${ }^{9}$, éstas se limitan a plasmarlas sin llegar a superposiciones de capas sobre diferentes temas para análisis espaciales más elaborados, lo que daría valor añadido a la explotación y visualización de datos a través de GIS ${ }^{10}$ (figura 3).

\section{Conclusiones}

GIS es una herramienta de uso potencial en muchos escenarios dentro del mundo de bibliotecas, archivos y centros de documentación. De forma general, la tecnología GIS puede facilitar la toma de decisiones en la gestión de las unidades de información (Venuda, 2005). Ayuda a la planificación y diseño de espacios económicamente sostenibles y capaces de proporcionar servicios de calidad, repercutiendo en una mejora de la gestión de los recursos y del servicio al usuario.

El desarrollo tecnológico actual de GIS y sus mejoras en sencillez de uso junto al abaratamiento de costes, hacen accesible su uso como herramienta de apoyo a las unidades de información. Con conocimientos GIS a nivel de usuario, existen ya pequeñas utilidades y programas que pueden ser empleados en centros para crear aplicaciones de valor añadido y toma de decisiones (Abresch et al., 2008).

Los GIS, solos o combinados, abren las puertas a numerosas formas de representar los datos de las unidades de información

Los datos disponibles en las unidades de información por sí solos, o en combinación con datos abiertos (open data) accesibles a través de la administración pública (FerrerSapena; Peset; Aleixandre-Benavent, 2011), pueden servir de partida para ser explotados y visualizados en GIS. En especial, los registros socio-económicos y demográficos de la población pueden ser útiles para segmentar un mercado y ofrecer un mejor servicio, o para compartir experiencias con otras unidades de información que den servicios a grupos demográficos similares (Hertel; Sprague, 2007). Los GIS solos o combinados abren las puertas a numerosas formas de representar los datos de las unidades de información.

Por otra parte se abren posibilidades para la aparición de nuevos perfiles profesionales especializados, tanto en la gestión de herramientas GIS en el día a día de las unidades de información, como, a un nivel más elevado de especialización, en un nuevo concepto de bibliotecas como centro de recursos GIS (Argentati, 1997).

Debido a la importancia que ha adquirido últimamente la dimensión geográfica, las herramientas GIS están logrando un fuerte protagonismo como tecnologías integradoras y vertebradoras de la información en el contexto geográfico, entre otros ámbitos, en el de las bibliotecas y unidades de información.

\section{Notas}

1.http://www.sciencedirect.com/science/journal/00991333/21/4

2. En las Jornadas Ibersid 2012 (Zaragoza, 1-4 de octubre), se ha incluido una sesión temática llamada "Información geográfica: gestión, acceso e interoperabilidad".

http://catedralogisman.unizar.es/news/detail.dot?id=7965

3. Se ha hecho una revisión de la bibliografía sobre GIS y bibliotecas más exhaustiva de la que se presenta aquí. En la tabla se reflejan solamente los trabajos más representativos o completos de cada una de las aplicaciones de GIS identificadas.

4. La biblioteca de la University of Virginia, desde hace casi 10 años dispone de un proyecto de este tipo, englobado dentro del Scholar's lab, y anteriormente conocido como Geostat Center, donde recoje materiales y recursos geoespaciales.

http://www2.lib.virginia.edu/scholarslab

\section{5. http://www.geolib.org/PLGDB.cfm}

6. Como muestra de manera tradicional de presentar los datos cienciométricos, la alianza 4 universidades ha publicado los datos de la actividad investigadora de las universidades españolas. Otro ejemplo serían los datos del Ranking de universidades españolas.

http://www.iune.es

http://www.rankinguniversidades.es

7. Un ejemplo relacionado es el Atlas nacional de España que permite la búsqueda, consulta, visualización, descarga y navegación de los diferentes mapas temáticos.

http://www.ign.es/siane/Principal.do

8. El concepto de inteligencia territorial viene definido por la Coordination Action of the European Network of Territorial Intelligence (Caenti), como el conocimiento que se necesita para entender las dinámicas y estructuras territoriales, así como los instrumentos usados para la generación y uso de este conocimiento para garantizar e impulsar el desarrollo territorial sostenible.

9. Sistema comunitario de información territorial, y mapa de conocimiento del sistema Madritd

http://www.c3it.uhu.es/atlas

http://www.madrimasd.org/informacionidi/mapaconocimiento/default.asp

10. La empresa de software GIS con más presencia a nivel mundial, ESRI, ha desarrollado algunos ejemplos específicos en diferentes contextos. En el caso de las bibliotecas muestran como ejemplo:

http://www.librosymapas.com

\section{Bibliografía}

Abresch, John; Hanson, Ardis; Heron, Susan-Jane; Reehling, Peter J. (2008). Integrating geographic information systems into libraries services: A guide for academic libraries. Hershey: IGI Global. ISBN: 9781599047263

Allen, Robert S. (2001). "Interdisciplinary research: a literature-based examination of disciplinary intersections using a common tool, geographic information system (GIS)". Scien- 
ce \& technology libraries, v. 21 n. 3-4, pp. 191-209. http://dx.doi.org/10.1300/J122v21n03_12

Argentati, Carolyn D. (1997). "Expanding horizons for GIS services in academic libraries". The journal of academic librarianship, v. 23, n. 6, pp. 463-468.

http://dx.doi.org/10.1016/S0099-1333(97)90170-1

Bishop, Bradley-Wade; Mandel, Lauren H. (2010). "Utilizing geographic information systems (GIS) in library research". Library hi tech, v. 28, n. 4, pp. 536-547.

http://dx.doi.org/10.1108/07378831011096213

Boxal, James (2003). "Geolibraries: geographers, librarians and spatial collaboration". The Canadian geographer, v. 47, n. 1, pp. 18-27.

http://dx.doi.org/10.1111/1541-0064.02e09

Castro-González, Carlos; González-Gallardo, Álvaro (2009). "Sistemas de información geográfica aplicados a bibliotecas móviles". En: IV Congreso Nacional de Bibliotecas Móviles. http://www.bibliotecaspublicas.info/biabp/acceso_ enlace?c_documento_id $=8627$

Coyle, Andrew (2011). "Interior library GIS". Library hi tech, v. 29 , n. 3, pp. 529-549.

http://dx.doi.org/10.1108/07378831111174468

Cox, Allan B.; Gifford, Fred (1997). "An overview to geographic information systems". The journal of academic librarianship, v. 23, n. 6, pp. 449-461.

http://dx.doi.org/10.1016/S0099-1333(97)90169-5

Ferrer-Sapena, Antonia; Peset, Fernanda; AleixandreBenavent, Rafael (2011). "Acceso a los datos públicos y su reutilización: open data y open government". El profesional de la información, v. 20, n. 3, pp. 260-269.

http://dx.doi.org/10.3145/epi.2011.may.03

Hawkins, Andrew M. (1994). "Geographical information systems (GIS): their use as decision support tools in public libraries and the integration of GIS with other computer technology". New library world, v. 95, n. 7, pp. 4-13. http://dx.doi.org/10.1108/03074809410070502

Hertel, Karen; Sprague, Nancy (2007). "GIS and census data: tools for library planning". Library hi tech, v. 25, n. 2, pp. 246-259. http://dx.doi.org/10.1108/07378830710755009

Koontz, Christie M.; Jue, Dean K.; McClure, Charles, R.; Bertot, John-Carlo (2004), "The public library geographic database: what can it do for your library?" Public libraries, v. 43, n. 2, pp. 113-118.

http://www.ala.org/pla/sites/ala.org.pla/files/content/ publications/publiclibraries/pastissues/43n2.pdf

Kowal, Kimberly C. (2002). "Tapping the web for GIS and mapping technologies: for all levels of libraries and users". Information technology and libraries, v. 21, n. 3, pp. 109114.

Michalec, Mychaelyn; Welsh, Teresa S. (2007). "Quantity and authorship of GIS articles in library and information science literature, 1990-2005". Science \& technology libraries, v. 27, n. 3, pp. 65-77.

http://dx.doi.org/10.1300/J122v27n03_05

Morris, Steven P. (2006). "Geospatial web services and geoarchiving: new opportunities and challenges in geographic information services". Library trends, v. 55, n. 2, pp. 285-303.

http://dx.doi.org/10.1353/lib.2006.0059

Neatrour, Anna; Morrow, Anne; Rockwell, Ken; Witkowski, Alan (2011). "Automating the production of map interfaces for digital collections using Google APIs". D-Lib magazine, v. 17, n. 9-10.

http://dx.doi.org/10.1045/september2011-neatrour

Preiser, Wolfgang F. E.; Wang, Xinhao (2006). "Assessing library performance with GIS and building evaluation methods". New library world, v. 107, n. 5-6, pp. 193-217. http://dx.doi.org/10.1108/03074800610665202

Venuda, Fabio (2005). "II GIS (geographic information system) in biblioteca". Bolletino AIB, v. 45, n. 3-4, pp. 327-346.

Xia, Jingfeng (2004). "Library space management: a GIS proposal". Library hi tech, v. 22, n. 4, pp. 375-382. http://dx.doi.org/10.1108/07378830410570476

Xia, Jingfeng (2005). "Visualizing occupancy of library study space with GIS maps". New library world, v. 106, n. 5-6, pp. 219-233.

http://dx.doi.org/10.1108/03074800510595832

\section{Suscripción EPI sólo online}

Pensando sobre todo en los posibles suscriptores latinoamericanos, ya no es obligatorio pagar la suscripción impresa de EPI para acceder a la online.

EPI se ofrece a instituciones en suscripción "sólo online" a un precio considerablemente más reducido $(96,69+21 \%$ IVA euros/año), puesto que en esta modalidad no hay que cubrir los gastos de imprenta ni de correo postal. 